\title{
New Approach to Intuitionistic Fuzzy Rough Sets
}

\author{
Sang Min Yun and Seok Jong Lee \\ Department of Mathematics, Chungbuk National University, Cheongju, Korea
}

\section{]jfis}

\begin{abstract}
The properties of the intuitionistic fuzzy rough sets are very complicated and inadequate in the sense of the extension of intuitionistic properties. In order to overcome this unnaturalness, we introduce a new definition of intuitionistic fuzzy rough sets and investigate important properties about the image and inverse image of an intuitionistic rough sets under a mapping. All the results obtained from this new definition are different from the results in other papers, and will be proven useful in expanding the related theory.
\end{abstract}

Keywords: Intuitionistic fuzzy topology, Intuitionistic fuzzy rough sets

\section{Introduction}

The notion of fuzzy sets was first introduced by Zadeh [1]. After that, many studies attempted to generalize the fuzzy set by using various approaches. Pawlak [2] introduced the concept of rough sets, Nanda and Majumda [3] and Coker [4] proposed the idea of fuzzy rough sets. Atanassov [5] introduced the idea of intuitionistic fuzzy sets. All these concepts provide useful means of expressing vagueness in real environments.

Combining the concepts of fuzzy rough sets and intuitionistic fuzzy sets, Samanta and Mondal [6] proposed the idea of intuitionistic fuzzy rough sets. By introducing further generalization, the authors [7, 8] also conducted a study on the intuitionistic fuzzy bitopology and intuitionistic smooth bitopology. Moreover, the categorical properties of the intuitionsitc fuzzy topological spaces were studied by the same research group [9-11].

Many attempts at combining fuzziness and roughness have been made. In [12], the measure

Received: Dec. 15, 2019

Revised : May 2, 2020

Accepted: May 5, 2020

Correspondence to: Seok Jong Lee (sjl@cbnu.ac.kr)

(CThe Korean Institute of Intelligent Systems

(c) This is an Open Access article distributed under the terms of the Creative Commons Attribution Non-Commercial License (http://creativecommons.org/licenses/ by-nc/3.0/] which permits unrestricted noncommercial use, distribution, and reproduction in any medium, provided the original work is properly cited. of fuzziness in rough sets is provided and studied. In [13] a general framework for the study of fuzzy rough sets is presented, in which both constructive and axiomatic approaches are made. Lower and upper approximations of intuitionistic fuzzy sets with respect to an intuitionistic fuzzy approximation space are first defined by Zhou et al. [14, 15]. Several important properties of intuitionistic fuzzy approximation operators are examined by many researchers including us [16-19].

However, the properties of the intuitionistic fuzzy rough sets are very complicated and inadequate in the sense of the extension of intuitionistic properties. This is because of the unnaturalness of the definition of fuzzy rough sets. For example, the double complement of a fuzzy rough set is different from itself. The property that the double complement of a set becomes the set itself is one of the essential properties of Boolean algebra. Hence this flaw is critical in expanding the related theory. In order to overcome this unnaturalness, we need a new approach to intuitionistic fuzzy rough sets. 
In this paper, we introduce a new definition of intuitionistic fuzzy rough sets and investigate important properties about the image and inverse image of an intuitionistic rough sets under a mapping. This new approach enables us to manipulate fuzzy rough sets more simply and easily. All the results obtained from this new definition are different from the results in other papers, and will be proven useful in expanding the related theory.

\section{Fuzzy Rough Sets}

In [3], the definition of fuzzy rough sets has been introduced. The paper said:

"we shall consider $(\mathcal{V}, \mathfrak{B})$ to be a rough universe where $\mathcal{V}$ is a nonempty set and $\mathfrak{B}$ is a Boolean subalgebra of the Boolean algebra of all subsets of $\mathcal{V}$. Also consider a rough set $X=\left(X_{L}, X_{U}\right) \in \mathfrak{B}^{2}$ with $X_{L} \subseteq X_{U}$. A fuzzy rough set in $X$ is an object of the form

$$
A=\left(A_{L}, A_{U}\right),
$$

where $A_{L}$ and $A_{U}$ are characterized by a pair of maps $A_{L}$ : $X_{L} \rightarrow L$ and $A_{U}: X_{U} \rightarrow L$ with $A_{L}(x) \leq A_{U}(x)$, for all $x \in X_{L}$, where $(L, \leq)$ is a fuzzy lattice."

Furthermore, the complement $\bar{A}$ of a fuzzy rough set $A=$ $\left(A_{L}, A_{U}\right)$ is defined by $(\bar{A})_{L}(x)=\left(A_{U>L}\right)^{\prime}(x), \forall x \in X_{L}$ and $(\bar{A})_{U}(x)=\left(A_{L<U}\right)^{\prime}(x), \forall x \in X_{U}$,

where $A_{U>L}(x)=A_{U}(x), \forall x \in X_{L}$ and

$$
A_{L<U}(x)= \begin{cases}A_{L}(x), & \text { if } x \in X_{L}, \\ \bigvee\left\{A_{L}(x) \mid x \in X_{L}\right\}, & \text { if } x \in X_{U}-X_{L}\end{cases}
$$

Unfortunately, the double complement of a fuzzy rough set $A$ is different from $A$, because $X_{L}$ and $X_{U}$ are different, i.e., $X_{L} \leq X_{U}$, The property that double complement of a set becomes the set itself is one of the essential properties of Boolean algebra. Hence this flaw is critical in expanding the related theory. Thus we are going to introduce the new definition of a fuzzy rough set by weakening the condition of the old definition. Then the properties we obtain in this paper are different from the results in the above paper.

Definition 2.1. Ler $X$ be an underlying set and $(L, \leq)$ a fuzzy lattice. A fuzzy rough set in $X$ is an object of the form

$$
A=\left(A_{L}, A_{U}\right),
$$

where $A_{L}$ and $A_{U}$ are defined by a pair of maps $A_{L}: X \rightarrow L$ and $A_{U}: X \rightarrow L$ with $A_{L}(x) \leq A_{U}(x)$, for all $x \in X$. Furthermore $\underline{0}=\left(\underline{0}_{L}, \underline{0}_{U}\right)$ the null fuzzy rough set and $\underline{1}=$ $\left(\underline{1}_{L}, \underline{1}_{U}\right)$ the whole fuzzy rough set in $X$,

Definition 2.2. For any two fuzzy rough sets $A=\left(A_{L}, A_{U}\right)$ and $B=\left(B_{L}, B_{U}\right)$ of $X$, we define the following:

(1) $A \subseteq B$ iff $A_{L}(x) \leq B_{L}(x)$ and $A_{U}(x) \leq B_{U}(x)$ for all $x \in X$.

(2) $A=B$ iff $A \subseteq B$ and $B \subseteq A$.

If $\left\{A_{i} \mid i \in J\right\}$ is a family of fuzzy rough sets in $X$, with $A_{i}=\left(\left(A_{i}\right)_{L},\left(A_{i}\right)_{U}\right)$, then we define the following:

(1) $\left(\bigcup_{i} A_{i}\right)_{L}(x)=\bigvee\left(A_{i}\right)_{L}(x)$ and $\left(\bigcup_{i} A_{i}\right)_{U}(x)=\bigvee\left(A_{i}\right)_{U}(x)$ for all $x \in X$.

(2) $\left(\bigcap_{i} A_{i}\right)_{L}(x)=\bigwedge\left(A_{i}\right)_{L}(x)$ and $\left(\bigcap_{i} A_{i}\right)_{U}(x)=\bigwedge\left(A_{i}\right)_{U}(x)$ for all $x \in X$.

Definition 2.3. The complement $\bar{A}=\left((\bar{A})_{L},(\bar{A})_{U}\right)$ of a fuzzy rough set $A=\left(A_{L}, A_{U}\right)$ in $X$ is defined by $(\bar{A})_{L}(x)=$ $\left(A_{U}(x)\right)^{\prime}$ and $(\bar{A})_{U}(x)=\left(A_{L}(x)\right)^{\prime}$ for all $x \in X$.

For any fuzzy rough set $A=\left(A_{L}, A_{U}\right)$ and for any $x \in X$,

$$
(\bar{A})_{L}(x)=\left(A_{U}(x)\right)^{\prime} \leq\left(A_{L}(x)\right)^{\prime}=(\bar{A})_{U}(x) .
$$

So, $\bar{A}$ is again a fuzzy rough set. Furthermore, for any fuzzy rough set $A=\left(A_{L}, A_{U}\right)$, we have the double complement property, i.e., $\overline{(\bar{A})}=A$.

Theorem 2.4. Let $A$ be any fuzzy rough set in $X$, then we have the following properties:

(1) $\underline{0} \subseteq A \subseteq \underline{1}$.

(2) $\overline{(\underline{0})}=\underline{1}, \overline{(\underline{1})}=\underline{0}$.

Theorem 2.5. If $A, B, C, D$ and $B_{i}, i \in J$ are fuzzy rough sets in $X$, then we have the following properties:

(1) $A \subseteq B$ and $C \subseteq D$ implies $A \cup C \subseteq B \cup D$ and $A \cap C \subseteq B \cap D$.

(2) $A \subseteq B$ and $B \subseteq C$ implies $A \subseteq C$.

(3) $A \cap B \subseteq A, B \subseteq A \cup B$.

(4) $A \cup\left(\bigcap_{i} B_{i}\right)=\bigcap_{i}\left(A \cup B_{i}\right), A \cap\left(\bigcup_{i} B_{i}\right)=\bigcup_{i}\left(A \cap B_{i}\right)$.

(5) $A \subseteq B$ implies $\bar{B} \subseteq \bar{A}$.

(6) $\overline{\bigcap_{i} B_{i}}=\bigcup_{i} \overline{B_{i}}, \overline{\bigcup_{i} B_{i}}=\bigcap_{i} \overline{B_{i}}$. 
Definition 2.6. Let $f: X \rightarrow Y$ be a mapping and $A=$ $\left(A_{L}, A_{U}\right)$ a fuzzy rough set in $X$. The image $f(A)=\left((f(A))_{L}\right.$, $\left.(f(A))_{U}\right)$ of $A$ under $f$ is defined by, for any $y \in Y$

$$
\begin{aligned}
(f(A))_{L}(y) & =f\left(A_{L}\right)(y) \\
& = \begin{cases}\underline{0}_{L}, & \text { if } f^{-1}(y)=\emptyset, \\
\bigvee\left\{A_{L}(x) \mid x \in f^{-1}(y)\right\}, & \text { otherwise, }\end{cases} \\
(f(A))_{U}(y) & =f\left(A_{U}\right)(y) \\
& = \begin{cases}\underline{0}_{U}, & \text { if } f^{-1}(y)=\emptyset, \\
\bigvee\left\{A_{U}(x) \mid x \in f^{-1}(y)\right\}, & \text { otherwise. }\end{cases}
\end{aligned}
$$

Let $B=\left(B_{L}, B_{U}\right)$ be a fuzzy rough set in $Y$. Then the inverse image $f^{-1}(B)=\left(\left(f^{-1}(B)\right)_{L},\left(f^{-1}(B)\right)_{U}\right)$ of $B$ under $f$ is defined by, for any $y \in Y$

$$
\begin{aligned}
& \left(f^{-1}(B)\right)_{L}(x)=f^{-1}\left(B_{L}\right)(x)=B_{L}(f(x)), \\
& \left(f^{-1}(B)\right)_{U}(x)=f^{-1}\left(B_{U}\right)(x)=B_{U}(f(x)) .
\end{aligned}
$$

The image of $A$ under $f$ is well-defined, because

$$
\begin{aligned}
& f\left(A_{L}\right)(y)=\bigvee\left\{A_{L}(x) \mid x \in f^{-1}(y)\right\} \\
& \leq \bigvee\left\{A_{U}(x) \mid x \in f^{-1}(y)\right\}=f\left(A_{U}\right)(y) .
\end{aligned}
$$

Moreover, the inverse image of $B$ under $f$ is well-defined, because

$$
f^{-1}\left(B_{L}\right)(x)=B_{L}(f(x)) \leq B_{U}(f(x))=f^{-1}\left(B_{U}\right)(x) .
$$

Theorem 2.7. If $f: X \rightarrow Y$ is a mapping, then for any fuzzy rough sets $A$ and $B$ in $X$, we have the following:

(1) $A \subseteq B$ implies $f(A) \subseteq f(B)$.

(2) If $f$ is surjective, then $f(\bar{A}) \supseteq \overline{f(A)}$.

(3) $f\left(\bigcup_{i \in J} A_{i}\right)=\bigcup_{i \in J} f\left(A_{i}\right)$.

(4) $f\left(\bigcap_{i \in J} A_{i}\right) \subseteq \bigcap_{i \in J} f\left(A_{i}\right)$.

Proof. (1) The proof is clear.

(2) Let $f$ be surjective.

$$
\begin{aligned}
(f(\bar{A}))_{L}(y) & =f\left((\bar{A})_{L}\right)(y) \\
& =\bigvee\left\{(\bar{A})_{L}(x) \mid x \in f^{-1}(y)\right\} \\
& =\bigvee\left\{\left(A_{U}(x)\right)^{\prime} \mid x \in f^{-1}(y)\right\} \\
& \geq \bigwedge\left\{\left(A_{U}(x)\right)^{\prime} \mid x \in f^{-1}(y)\right\}
\end{aligned}
$$

$$
\begin{aligned}
& =\left(\bigvee\left\{\left(A_{U}(x)\right) \mid x \in f^{-1}(y)\right\}\right)^{\prime} \\
& =\left(f\left(A_{U}\right)(y)\right)^{\prime}=\left((f(A))_{U}(y)\right)^{\prime} \\
& =(\overline{f(A)})_{L}(y) .
\end{aligned}
$$

The proof of the upper part is similar.

$$
\begin{aligned}
\left(f\left(\bigcup_{i \in J} A_{i}\right)\right)_{L}(y) & =f\left(\left(\bigcup_{i \in J} A_{i}\right)_{L}\right)(y) \\
& =\bigvee\left\{\left(\bigcup_{i \in J} A_{i}\right)_{L}(x) \mid x \in f^{-1}(y)\right\} \\
& =\bigvee\left\{\bigvee_{i \in J}\left(A_{i}\right)_{L}(x) \mid x \in f^{-1}(y)\right\} \\
& =\bigvee_{i \in J}\left\{\bigvee_{x \in f^{-1}(y)}\left(A_{i}\right)_{L}(x)\right\} \\
& =\bigvee_{i \in J}\left\{f\left(\left(A_{i}\right)_{L}\right)(y)\right\} \\
& =\bigcup_{i \in J}\left(f\left(A_{i}\right)\right)_{L}(y) .
\end{aligned}
$$

The proof of the upper part is similar.

$$
\begin{aligned}
\left(f\left(\bigcap_{i \in J} A_{i}\right)\right)_{L}(y) & =f\left(\left(\bigcap_{i \in J} A_{i}\right)_{L}\right)(y) \\
& =\bigvee\left\{\left(\bigcap_{i \in J} A_{i}\right)_{L}(x) \mid x \in f^{-1}(y)\right\} \\
& =\bigvee\left\{\bigwedge_{i \in J}\left(A_{i}\right)_{L}(x) \mid x \in f^{-1}(y)\right\} \\
& \leq \bigwedge_{i \in J}\left\{\bigvee_{x \in f^{-1}(y)}\left(A_{i}\right)_{L}(x)\right\} \\
& =\bigwedge_{i \in J}\left\{f\left(\left(A_{i}\right)_{L}\right)(y)\right\} \\
& =\bigcap_{i \in J}\left(f\left(A_{i}\right)\right)_{L}(y) .
\end{aligned}
$$

The proof of the upper part is similar.

The surjectiveness is essential in (2) of the above theorem. It can be shown by the following example.

Example 2.8. Let $X=\{1,2,3\}, Y=\{a, b, c\}$. Let $f: X \rightarrow$ $Y$ be a mapping with $f(1)=a, f(2)=f(3)=c$. Then $f$ is not surjective. Consider a fuzzy rough set

$$
A=(\{1 / 0.4,2 / 0.3\},\{1 / 0.4,2 / 0.4\}) .
$$

Then

$$
\bar{A}=(\{1 / 0.6,2 / 0.6\},\{1 / 0.6,2 / 0.7\}) .
$$




$$
f(\bar{A})=(\{a / 0.6, b / 0, c / 0.6\},\{a / 0.6, b / 0, c / 0.7\}) .
$$

But

$$
f(A)=(\{a / 0.4, b / 0, c / 0.3\},\{a / 0.4, b / 0, c / 0.4\}),
$$

and so

$$
\overline{f(A)}=(\{a / 0.6, b / 1, c / 0.6\},\{a / 0.6, b / 1, c / 0.7\}) .
$$

Hence $f(\bar{A}) \subseteq \overline{f(A)}$.

Remark 2.9. If $f: X \rightarrow Y$ and $g: Y \rightarrow Z$ are mappings, then for any fuzzy rough set $C$ in $Z$, the inverse image of $C$ under $g \circ f$ is defined by $(g \circ f)^{-1}(C)=f^{-1}\left(g^{-1}(C)\right)$ where $g \circ f$ is the composition of $g$ and $f$.

Theorem 2.10. If $f: X \rightarrow Y$ is a mapping, then for all fuzzy rough sets $B, C, B_{i}, i \in J$ in $Y$, we have the following:

(1) $f^{-1}(\bar{B})=\overline{f^{-1}(B)}$.

(2) $B \subseteq C$ implies $f^{-1}(B) \subseteq f^{-1}(C)$.

(3) $f^{-1}\left(\bigcap_{i} B_{i}\right)=\bigcap_{i} f^{-1}\left(B_{i}\right)$.

(4) $f^{-1}\left(\bigcup_{i} B_{i}\right)=\bigcup_{i} f^{-1}\left(B_{i}\right)$.

Proof. (1) $\left(f^{-1}(\bar{B})\right)_{L}(x)=f^{-1}\left((\bar{B})_{L}\right)(x)=(\bar{B})_{L}(f(x))$ $=\left(B_{U}(f(x))\right)^{\prime}=\left(f^{-1}\left(B_{U}\right)(x)\right)^{\prime}=\left(\left(f^{-1}(B)\right)_{U}(x)\right)^{\prime}=$ $\left(\overline{f^{-1}(B)}\right)_{L}(x)$. The proof of upper part is similar. Hence $f^{-1}(\bar{B})=\overline{f^{-1}(B)}$.

(2) Since $B \subseteq C, B_{L}(y) \leq C_{L}(y)$ for all $y \in Y$. $\left(f^{-1}(B)\right)_{L}$ $(x)=f^{-1}\left(B_{L}\right)(x)=B_{L}(f(x)) \leq C_{L}(f(x))=f^{-1}\left(C_{L}\right)(x)$ $=\left(f^{-1}(C)\right)_{L}(x)$. The proof of upper part is similar. Hence $B \subseteq C$ implies $f^{-1}(B) \subseteq f^{-1}(C)$.

(3) $\left(f^{-1}\left(\bigcap_{i} B_{i}\right)\right)_{L}(x)=f^{-1}\left(\left(\bigcap_{i} B_{i}\right)_{L}\right)(x)=\left(\bigcap_{i} B_{i}\right)_{L}$ $(f(x))=\bigwedge_{i}\left(B_{i}\right)_{L}(f(x))=\bigwedge_{i} f^{-1}\left(\left(B_{i}\right)_{L}\right)(x)=\bigwedge_{i}\left(f^{-1}\right.$ $\left.\left(B_{i}\right)\right)_{L}(x)=\left(\bigcap_{i} f^{-1}\left(B_{i}\right)\right)_{L}(x)$. The proof of upper part is similar. Hence $f^{-1}\left(\bigcap_{i} B_{i}\right)=\bigcap_{i} f^{-1}\left(B_{i}\right)$.

(4) Similarly.

Theorem 2.11. If $f: X \rightarrow Y$ is a mapping, then for any fuzzy rough set $A$ in $X$ and $B$ in $Y$, we have the following:

(1) $B \supseteq f\left(f^{-1}(B)\right)$.

(2) $A \subseteq f^{-1}(f(A))$.

\section{Intuitionistic Fuzzy Rough Sets}

Definition 3.1. If $A=\left(A_{L}, A_{U}\right)$ and $B=\left(B_{L}, B_{U}\right)$ are two fuzzy rough sets in $X$ with $B \subseteq \bar{A}$, then the ordered pair $(A, B)$ is called an intuitionistic fuzzy rough set (briefly IF rough set) in $X$. The condition $B \subseteq \bar{A}$ is called the intuitionistic condition (briefly IC).

Remark 3.2. In [6], the IC is that $B \subseteq \bar{A}$ and $A \subseteq \bar{B}$. But by the definition of IF rough set in this paper, we get the followings. If $B \subseteq \bar{A}$, then $B_{L}(x) \leq(\bar{A})_{L}(x)=\left(A_{U}(x)\right)^{\prime}$. i.e. $B_{L}(x) \leq$ $\left(A_{U}(x)\right)^{\prime}$ and $B_{U}(x) \leq\left(A_{L}(x)\right)^{\prime}$. So $A_{U}(x) \leq\left(B_{L}(x)\right)^{\prime}$ and $A_{L}(x) \leq\left(B_{U}(x)\right)^{\prime}$. Thus $A \subseteq \bar{B}$. Moreover, the converse is clear. Therefore $B \subseteq \bar{A}$ iff $A \subseteq \bar{B}$.

Definition 3.3. $0^{*}=(\underline{0}, \underline{1})$ and $1^{*}=(\underline{1}, \underline{0})$ are called the null IF rough set and the whole IF rough set in $X$, respectively. Clearly $\overline{0^{*}}=1^{*}$ and $\overline{1^{*}}=0^{*}$.

We denote by IFRS(X) the collection of all IF rough sets in $X$. Usually we shall use letters $X, Y, Z, \ldots$ to denote sets, letters $A, B, C, \ldots$ to denote fuzzy rough sets, and letters $P, Q, R, \ldots$ to denote IF rough sets.

Definition 3.4. 53.4 Let $P=(A, B)=\left(\left(A_{L}, A_{U}\right),\left(B_{L}, B_{U}\right)\right)$ and $Q=(C, D)=\left(\left(C_{L}, C_{U}\right),\left(D_{L}, D_{U}\right)\right)$ be two IF rough sets in $X$. We define the following:

(1) $P \subseteq Q$ iff $A \subseteq C$ and $B \supseteq D$.

(2) $P=Q$ iff $P \subseteq Q$ and $P \supseteq Q$.

(3) The complement $\bar{P}$ of $P=(A, B)$ in $X$, is defined by $\bar{P}=(B, A)$.

(4) For IF rough sets $P_{i}=\left(A_{i}, B_{i}\right)$ in $X, i \in J$, we define

$$
\begin{aligned}
& \bigcup_{i \in J} P_{i}=\left(\bigcup_{i \in J} A_{i}, \bigcap_{i \in J} B_{i}\right), \\
& \bigcap_{i \in J} P_{i}=\left(\bigcap_{i \in J} A_{i}, \bigcup_{i \in J} B_{i}\right) .
\end{aligned}
$$

Remark 3.5. Let $P_{i}=\left(A_{i}, B_{i}\right)$ be an IF rough set for all $i \in \Delta$, then $A_{i} \subseteq \overline{B_{i}}$ for all $i \in \Delta$. For any $k \in \Delta$, we have

$$
A_{k} \subseteq \overline{B_{k}} \subseteq \bigcup_{i \in \Delta} \overline{B_{i}}=\overline{\bigcap_{i \in \Delta} B_{i}} .
$$

Thus $\bigcup_{i \in \Delta} A_{i} \subseteq \overline{\bigcap_{i \in \Delta} B_{i}}$. Hence $\bigcup_{i \in \Delta} P_{i}=\left(\bigcup_{i \in \Delta} A_{i}, \bigcap_{i \in \Delta} B_{i}\right)$ is also an IF rough set. 
Similarly, for any $k \in \Delta, \bigcap_{i} A_{i} \subseteq A_{k} \subseteq \overline{B_{k}}$. So $\bigcap_{i} A_{i} \subseteq$ $\bigcap_{i} \overline{B_{i}}=\overline{\bigcup_{i} B_{i}}$. Hence $\bigcap_{i} P_{i}=\left(\bigcap_{i} A_{i}, \bigcup_{i} B_{i}\right)$ is also an IF rough set.

Theorem 3.6. Let $P=(A, B), Q=(C, D), R=(E, F)$ and $P_{i}=\left(A_{i}, B_{i}\right), i \in J$ be IF rough sets in $X$, then we have the following:

(1) $P \cap P=P=P \cup P$.

(2) $P \cap Q=Q \cap P, P \cup Q=Q \cup P$

(3) $(P \cap Q) \cap R=P \cap(Q \cap R),(P \cup Q) \cup R=P \cup(Q \cup R)$.

(4) $P \cap Q \subseteq P, Q \subseteq P \cup Q$.

(5) $P \subseteq Q$ and $Q \subseteq R$ implies $P \subseteq R$.

(6) $P_{i} \subseteq Q, \forall i \in J$ implies $\bigcup_{i} P_{i} \subseteq Q$.

(7) $Q \subseteq P_{i}, \forall i \in J$ implies $Q \subseteq \bigcap_{i} P_{i}$.

(8) $Q \cup\left(\bigcap_{i} P_{i}\right)=\bigcap_{i}\left(Q \cup P_{i}\right)$.

(9) $Q \cap\left(\bigcup_{i} P_{i}\right)=\bigcup_{i}\left(Q \cap P_{i}\right)$.

(10) $\overline{(\bar{P})}=P$.

(11) $P \subseteq Q$ iff $\bar{Q} \subseteq \bar{P}$.

(12) $\overline{\bigcup_{i} P_{i}}=\bigcap_{i} \overline{P_{i}}, \overline{\bigcap_{i} P_{i}}=\bigcup_{i} \overline{P_{i}}$.

Proof. (4) $P \cap Q=(A \cap C, B \cup D) \subseteq(A, B) \subseteq(A \cup C, B \cap$ $D)=P \cup Q$.

(12) $\overline{\bigcup_{i} P_{i}}=\overline{\left(\bigcup_{i} A_{i}, \bigcap_{i} B_{i}\right)}=\left(\bigcap_{i} B_{i}, \bigcup_{i} A_{i}\right)=\bigcap_{i}\left(B_{i}, A_{i}\right)$ $=\bigcap_{i} \overline{P_{i}}$.

Definition 3.7. Let $f: X \rightarrow Y$ be a mapping. Let $P=$ $(A, B)=\left(\left(A_{L}, A_{U}\right),\left(B_{L}, B_{U}\right)\right)$ be an IF rough set in $X$. Then we define an image of $P$ under $f$ by

$$
f(P)=(f(A), \overline{f(\bar{B})}),
$$

where $f(A)=\left(f\left(A_{L}\right), f\left(A_{U}\right)\right)$ and $\overline{f(\bar{B})}=\left((\overline{f(\bar{B})})_{L}\right.$, $\left.(\overline{f(\bar{B})})_{U}\right)$ is defined by $(\overline{f(\bar{B})})_{L}=\overline{f\left((\bar{B})_{L}\right)}$ and $(\overline{f(\bar{B})})_{U}=$ $\overline{f\left((\bar{B})_{U}\right)}$.

We already know that $f(A)(y)=\left(f\left(A_{L}\right)(y), f\left(A_{U}\right)(y)\right)=$ $\left(\bigvee\left\{A_{L}(x) \mid x \in f^{-1}(y)\right\}, \bigvee\left\{A_{U}(x) \mid x \in f^{-1}(y)\right\}\right)$ for any fuzzy rough set $A$ in $X$ and a function $f: X \rightarrow Y$.

Theorem 3.8. By the above definition, we have the following conclusion:

For any fuzzy rough set $B$ in $X$ and a function $f: X \rightarrow Y$, We have $\overline{f(\bar{B})}(y)=\left(\bigwedge\left\{\left(B_{L}(x)\right) \mid x \in f^{-1}(y)\right\}, \bigwedge\left\{\left(B_{U}(x)\right) \mid\right.\right.$ $\left.\left.x \in f^{-1}(y)\right\}\right)$.
Proof.

$$
\begin{aligned}
f\left((\bar{B})_{L}\right)(y) & =\bigvee\left\{(\bar{B})_{L}(x) \mid x \in f^{-1}(y)\right\} \\
& =\bigvee\left\{\left(B_{U}(x)\right)^{\prime} \mid x \in f^{-1}(y)\right\} \\
& =\left(\bigwedge\left\{\left(B_{U}(x)\right) \mid x \in f^{-1}(y)\right\}\right)^{\prime}, \\
f\left((\bar{B})_{U}\right)(y) & =\bigvee\left\{(\bar{B})_{U}(x) \mid x \in f^{-1}(y)\right\} \\
& =\bigvee\left\{\left(B_{L}(x)\right)^{\prime} \mid x \in f^{-1}(y)\right\} \\
& =\left(\bigwedge\left\{\left(B_{L}(x)\right) \mid x \in f^{-1}(y)\right\}\right)^{\prime} .
\end{aligned}
$$

So, $f(\bar{B})(y)=\left(\left(\bigwedge\left\{\left(B_{U}(x)\right) \mid x \in f^{-1}(y)\right\}\right)^{\prime},\left(\bigwedge\left\{\left(B_{L}(x)\right) \mid\right.\right.\right.$ $\left.\left.\left.x \in f^{-1}(y)\right\}\right)^{\prime}\right)$. Thus, $\overline{f(\bar{B})}(y)=\left(\bigwedge\left\{\left(B_{L}(x)\right) \mid x \in\right.\right.$ $\left.\left.f^{-1}(y)\right\}, \bigwedge\left\{\left(B_{U}(x)\right) \mid x \in f^{-1}(y)\right\}\right)$.

Remark 3.9. We will prove that the image of any IF rough set $P=(A, B)$ satisfies the IC.

$$
\begin{aligned}
(f(A))_{L}(y) & =f\left(A_{L}\right)(y) \\
& =\bigvee\left\{A_{L}(x) \mid x \in f^{-1}(y)\right\} \\
& \leq \bigvee\left\{(\bar{B})_{L}(x) \mid x \in f^{-1}(y)\right\} \\
& =\bigvee\left\{\left(B_{U}(x)\right)^{\prime} \mid x \in f^{-1}(y)\right\} \\
& =\left(\bigwedge\left\{\left(B_{U}(x)\right) \mid x \in f^{-1}(y)\right\}\right)^{\prime} \\
& =\left((\overline{f(\bar{B})})_{U}(y)\right)^{\prime} \\
& =(\overline{(\overline{f(\bar{B})})})_{L}(y) .
\end{aligned}
$$

The proof of the upper part is similar. So, if $P=(A, B)$ be an IF rough set in $X$, then $f(P)=(f(A), \overline{f(\bar{B})})$ is also an IF rough set in $Y$.

Remark 3.10. By the above theorem, we have the following conclusion:

For any IF rough set $P=(A, B)$ in $X$ and any map $f$ : $X \rightarrow Y$, The image $f(P)=(f(A), f(B))$ of $P$ under $f$ is actually expressed as follows:

For any $y \in Y$

$f(P)(y)=\left(\bigvee\left\{A(x) \mid x \in f^{-1}(y)\right\}, \bigwedge\left\{B(x) \mid x \in f^{-1}(y)\right\}\right)$,

where

$\bigvee\left\{A(x) \mid x \in f^{-1}(y)\right\}$

$=\left\{\begin{array}{l}\underline{0}, \quad \text { if } f^{-1}(y)=\emptyset, \\ \left(\bigvee\left\{A_{L}(x) \mid x \in f^{-1}(y)\right\}, \bigvee\left\{A_{U}(x) \mid x \in f^{-1}(y)\right\}\right), \\ \text { if } f^{-1}(y) \neq \emptyset,\end{array}\right.$ 


$$
\begin{aligned}
& \bigwedge\left\{B(x) \mid x \in f^{-1}(y)\right\} \\
& =\left\{\begin{array}{l}
\underline{1}, \quad \text { if } f^{-1}(y)=\emptyset, \\
\left(\bigwedge\left\{B_{L}(x) \mid x \in f^{-1}(y)\right\}, \bigwedge\left\{B_{U}(x) \mid x \in f^{-1}(y)\right\}\right), \\
\text { if } f^{-1}(y) \neq \emptyset .
\end{array}\right.
\end{aligned}
$$

Definition 3.11. Let $f: X \rightarrow Y$ be a mapping and $Q=$ $(C, D)=\left(\left(C_{L}, C_{U}\right),\left(D_{L}, D_{U}\right)\right)$ an IF rough set in $Y$. Then we define an inverse image of $Q$ under $f$ by

$$
f^{-1}(Q)=\left(f^{-1}(C), f^{-1}(D)\right)
$$

where $f^{-1}(C)=\left(\left(f^{-1}(C)\right)_{L},\left(f^{-1}(C)\right)_{U}\right)=\left(f^{-1}\left(C_{L}\right)\right.$, $\left.f^{-1}\left(C_{U}\right)\right)$ and $f^{-1}(D)=\left(\left(f^{-1}(D)\right)_{L},\left(f^{-1}(D)\right)_{U}\right)=\left(f^{-1}\right.$ $\left.\left(D_{L}\right), f^{-1}\left(D_{U}\right)\right)$.

Remark 3.12. $f^{-1}\left(C_{L}\right)(x)=C_{L}(f(x)) \leq(\bar{D})_{L}(f(x))=$ $f^{-1}\left((\bar{D})_{L}\right)(x)=\left(\overline{f^{-1}(D)}\right)_{L}(x)$. The upper part is also hold. Hence, the inverse image of an IF rough set under $f$ is also an IF rough set.

Theorem 3.13. Let $f: X \rightarrow Y$ be a mapping. For any IF rough set $P$ and $Q$ on $X$, we have the following:

(1) $P \subseteq Q$ implies $f(P) \subseteq f(Q)$.

(2) If $f$ is surjective, then $f(\bar{P}) \supseteq \overline{f(P)}$.

Proof. (1) It is clear.

(2) Let $P=(A, B)$ be an IF rough set. Then $f(\bar{P})=$ $f((B, A))=(f(B), \overline{f(\bar{A})})$. Since $f(P)=f((A, B))=$ $(f(A), \overline{f(\bar{B})}), \overline{f(P)}=(\overline{f(\bar{B})}, f(A))$. Consider $(f(B))_{L}(y)=$ $f\left(B_{L}\right)(y)=\bigvee\left\{B_{L}(x) \mid x \in f^{-1}(y)\right\} \geq \bigwedge\left\{B_{L}(x) \mid x \in\right.$ $\left.f^{-1}(y)\right\}=(\overline{f(\bar{B})})_{L}(y)$. And the proof of the upper part is similar. Thus $f(B) \supseteq \overline{f(\bar{B})}$. Similarly, $f(A) \supseteq \overline{f(\bar{A})}$. Therefore $f(\bar{P})=(f(B), \overline{f(\bar{A})}) \supseteq(\overline{f(\bar{B})}, f(A))=\overline{f(P)}$.

Definition 3.14. If $f: X \rightarrow Y$ and $g: Y \rightarrow Z$ are mappings, then the inverse image of IF rough set $W$ in $Z$ under $(g \circ f)$ is defined by $(g \circ f)^{-1}(W)=f^{-1}\left(g^{-1}(W)\right)$ where $g \circ f$ is the composition of $g$ and $f$.

Theorem 3.15. Let $f: X \rightarrow Y$ be a mapping. For any IF rough set $R, S$ and $R_{i}, i \in J$ in $Y$, we have the following:

(1) $f^{-1}(\bar{R})=\overline{f^{-1}(R)}$.

(2) $R \subseteq S$ implies $f^{-1}(R) \subseteq f^{-1}(S)$.

(3) $f^{-1}\left(\bigcup_{i} R_{i}\right)=\bigcup_{i} f^{-1}\left(R_{i}\right)$.
(4) $f^{-1}\left(\bigcap_{i} R_{i}\right)=\bigcap_{i} f^{-1}\left(R_{i}\right)$.

Proof. (1) Let $R=(A, B)$, then $\bar{R}=(B, A) \cdot f^{-1}(\bar{R})=$ $\left(f^{-1}(B), f^{-1}(A)\right)=\overline{\left(f^{-1}(A), f^{-1}(B)\right)}=\overline{f^{-1}(R)}$.

(3) Let $R_{i}=\left(A_{i}, B_{i}\right)$ for all $i \in J$, then $\bigcup_{i} R_{i}=\bigcup_{i}\left(A_{i}, B_{i}\right)$ $=\left(\bigcup_{i} A_{i}, \bigcap_{i} B_{i}\right) \cdot f^{-1}\left(\bigcup_{i} R_{i}\right)=f^{-1}\left(\left(\bigcup_{i} A_{i}, \bigcap_{i} B_{i}\right)\right)=$ $\left(f^{-1}\left(\bigcup_{i} A_{i}\right), f^{-1}\left(\bigcap_{i} B_{i}\right)\right)=\left(\bigcup_{i} f^{-1}\left(A_{i}\right), \bigcap_{i} f^{-1}\left(B_{i}\right)\right)=$ $\bigcup_{i}\left(f^{-1}\left(A_{i}\right), f^{-1}\left(B_{i}\right)\right)=\bigcup_{i} f^{-1}\left(R_{i}\right)$.

Theorem 3.16. Let $f: X \rightarrow Y$ be a mapping. For any IF rough set $P$ in $X$ and $R$ in $Y$, we have the following:

(1) $R \supseteq f\left(f^{-1}(R)\right)$.

(2) $P \subseteq f^{-1}(f(P))$.

Proof. (1) Let $R=(C, D)$ be an IF rough set for some fuzzy rough sets $C, D$. Then $f^{-1}(R)=\left(f^{-1}(C), f^{-1}(D)\right)$. We have

$$
\begin{aligned}
& f\left(f^{-1}(R)\right)(y) \\
& =\left(\bigvee\left\{f^{-1}(C)(x)\right\}, \bigwedge\left\{f^{-1}(D)(x)\right\}\right) \text { for all } x \in f^{-1}(y) \\
& = \begin{cases}(\underline{0}, \underline{1}), \quad \text { if } f^{-1}(y)=\emptyset, \\
(\bigvee\{C(f(x))\}, \bigwedge\{D(f(x))\}) \text { for all } x \in f^{-1}(y), \\
\text { if } f^{-1}(y) \neq \emptyset\end{cases} \\
& \leq(C(y), D(y)) \\
& =R(y) .
\end{aligned}
$$

Hence $R \supseteq f\left(f^{-1}(R)\right)$.

(2) Let $P=(A, B)$ be an IF rough set. Then

$$
\begin{aligned}
& f^{-1}(f(P))(x) \\
& =f(P)(f(x))=f((A, B))(f(x)) \\
& =\left(\bigvee\left\{A(t) \mid t \in f^{-1}(f(x))\right\}, \bigwedge\left\{B(t) \mid t \in f^{-1}(f(x))\right\}\right) \\
& \geq(A(x), B(x)) \\
& =P(x) .
\end{aligned}
$$

Hence $P \subseteq f^{-1}(f(P))$.

In general, the equality does not hold in the above theorem. It can be shown by the following two examples.

Example 3.17. Let $X=\{1,2,3\}, Y=\{a, b, c\}$. And let $f$ : $X \rightarrow Y$ be a mapping with $f(1)=a, f(2)=f(3)=c$. Consider an IF rough set $R=(C, D)=((\{a / 0.4, b / 0.3\},\{a / 0.4$, $b / 0.4\}),(\{a / 0.2, b / 0.4\},\{a / 0.3, b / 0.4\}))$. Then we have $R(b)$ $=((0.3,0.4),(0.4,0.4))$. But $f\left(f^{-1}(R)\right)(b)=((0,1),(1,0))$. Thus $R \neq f\left(f^{-1}(R)\right)$. 
Example 3.18. Let $X=\{1,2,3\}, Y=\{a, b, c\}$. And let $f$ : $X \rightarrow Y$ be a mapping with $f(1)=a, f(2)=f(3)=c$. Consider an IF rough set $P=(A, B)=((\{1 / 0.4,2 / 0.3\},\{1 / 0.4$, $2 / 0.4\}),(\{1 / 0.2,2 / 0.4\},\{1 / 0.3,2 / 0.4\}))$. Then we have $P(3)$ $=((0,1),(1,0))$. But $f^{-1}(f(P))(3)=((0.3,0.4),(0.4,0.4))$. Thus $P \neq f^{-1}(f(P))$.

Theorem 3.19. Let $f: X \rightarrow Y$ be a mapping. For any IF rough sets $P, Q$ in $X$, we have $f(P \cup Q)=f(P) \cup f(Q)$.

Proof. Let $P=(A, B), Q=(C, D)$ be two IF rough sets.

$$
\begin{aligned}
f & (P \cup Q)(y) \\
= & f((A, B) \cup(C, D))(y) \\
= & f((A \cup C, B \cap D))(y) \\
= & (\bigvee\{(A \cup C)(x)\}, \bigwedge\{(B \cap D)(x)\}) \text { for all } x \in f^{-1}(y) \\
= & (\bigvee\{A(x) \vee C(x)\}, \bigwedge\{B(x) \wedge D(x)\}) \text { for all } x \in f^{-1}(y) \\
= & \left(\left(\bigvee\left\{A(x) \mid x \in f^{-1}(y)\right\}\right) \vee\left(\bigvee\left\{C(x) \mid x \in f^{-1}(y)\right\}\right),\right. \\
& \left.\left(\bigwedge\left\{B(x) \mid x \in f^{-1}(y)\right\}\right) \wedge\left(\bigwedge\left\{D(x) \mid x \in f^{-1}(y)\right\}\right)\right) \\
= & \left(\bigvee\left\{A(x) \mid x \in f^{-1}(y)\right\}, \bigwedge\left\{B(x) \mid x \in f^{-1}(y)\right\}\right) \\
& \vee\left(\bigvee\left\{C(x) \mid x \in f^{-1}(y)\right\}, \bigwedge\left\{D(x) \mid x \in f^{-1}(y)\right\}\right) \\
= & f(P)(y) \vee f(Q)(y) \\
= & (f(P) \cup f(Q))(y) .
\end{aligned}
$$

Corollary 3.20. If $P_{1}, P_{2}, \cdots, P_{n}$ are IF rough sets in $X$, then

$$
f\left(P_{1} \cup P_{2} \cup \cdots \cup P_{n}\right)=f\left(P_{1}\right) \cup f\left(P_{2}\right) \cup \cdots \cup f\left(P_{n}\right) .
$$

Theorem 3.21. Let $f: X \rightarrow Y$ be a mapping. For any IF rough sets $P, Q$ in $X$, we have $f(P \cap Q) \subseteq f(P) \cap f(Q)$.

Proof. Let $P=(A, B), Q=(C, D)$ for some fuzzy rough sets $A, B, C$ and $D$.

$$
\begin{aligned}
f & (P \cap Q)(y) \\
= & f((A, B) \cap(C, D))(y) \\
= & f((A \cap C, B \cup D))(y) \\
= & (\bigvee\{(A \cap C)(x)\}, \bigwedge\{(B \cup D)(x)\}) \text { for all } x \in f^{-1}(y) \\
= & (\bigvee\{A(x) \wedge C(x)\}, \bigwedge\{B(x) \bigvee D(x)\}) \text { for all } x \in f^{-1}(y) \\
\leq & \left(\left(\bigvee\left\{A(x) \mid x \in f^{-1}(y)\right\}\right) \wedge\left(\bigvee\left\{C(x) \mid x \in f^{-1}(y)\right\}\right)\right. \\
& \left.\left(\bigwedge\left\{B(x) \mid x \in f^{-1}(y)\right\}\right) \vee\left(\bigwedge\left\{D(x) \mid x \in f^{-1}(y)\right\}\right)\right) \\
= & \left(\bigvee\left\{A(x) \mid x \in f^{-1}(y)\right\}, \bigwedge\left\{B(x) \mid x \in f^{-1}(y)\right\}\right) \\
& \wedge\left(\bigvee\left\{C(x) \mid x \in f^{-1}(y)\right\}, \bigwedge\left\{D(x) \mid x \in f^{-1}(y)\right\}\right)
\end{aligned}
$$

$$
\begin{aligned}
& =f(P)(y) \wedge f(Q)(y) \\
& =(f(P) \cap f(Q))(y) .
\end{aligned}
$$

Hence $f(P \cap Q) \subseteq f(P) \cap f(Q)$.

Corollary 3.22. If $f: X \rightarrow Y$ is one-one, then clearly $f(P \cap$ $Q)=f(P) \cap f(Q)$.

But in general $f(P \cap Q) \neq f(P) \cap f(Q)$, which can be shown by the following example.

Example 3.23. Let $X=\{x, y\}$ and $Y=\{a\}$ and a mapping $f: X \rightarrow Y$ be defined by $f(x)=f(y)=a$. Let $P=((\{x / 0.4, y / 0.3\},\{x / 0.4, y / 0.4\}),(\{x / 0.2, y / 0.4\}$, $\{x / 0.3, y / 0.4\}))$, and $Q=((\{x / 0.3, y / 0.4\},\{x / 0.3, y / 0.4\})$, $(\{x / 0.3, y / 0.2\},\{x / 0.3, y / 0.3\}))$. Clearly $P, Q$ are IF rough sets in $X$. And $P \cap Q=((\{x / 0.3, y / 0.3\},\{x / 0.3, y / 0.4\})$, $(\{x / 0.3, y / 0.4\},\{x / 0.3, y / 0.4\}))$.

Thus

$$
\begin{aligned}
& f(P \cap Q)=((\{a / 0.3\},\{a / 0.4\}),(\{a / 0.3\},\{a / 0.3\})) \\
& f(P)=((\{a / 0.4\},\{a / 0.4\}),(\{a / 0.2\},\{a / 0.3\})) \\
& f(Q)=((\{a / 0.4\},\{a / 0.4\}),(\{a / 0.2\},\{a / 0.3\}))
\end{aligned}
$$

So $f(P) \cap f(Q)=((\{a / 0.4\},\{a / 0.4\}),(\{a / 0.2\},\{a / 0.3\}))$. Hence $f(P \cap Q) \neq f(P) \cap f(Q)$.

Theorem 3.24. Let $f: X \rightarrow Y$ be a mapping. For any IF rough set $P_{i}, i \in J$, we have the following:

(1) $f\left(\bigcup_{i \in J} P_{i}\right)=\bigcup_{i \in J}\left(P_{i}\right)$.

(2) $f\left(\bigcap_{i \in J} P_{i}\right) \subseteq \bigcap_{i \in J}\left(P_{i}\right)$.

Proof. (1) Let $P_{i}=\left(A_{i}, B_{i}\right)$ where $A_{i}, B_{i}$ are fuzzy rough sets for all $i \in J$. Then

$$
\begin{aligned}
& f\left(\bigcup_{i \in J} P_{i}\right)(y) \\
& =f\left(\left(\bigcup_{i \in J} A_{i}, \bigcap_{i \in J} B_{i}\right)\right)(y)=\left(f\left(\bigcup_{i \in J} A_{i}\right)(y), \overline{f\left(\overline{\bigcap_{i \in J} B_{i}}\right)}(y)\right) \\
& =\left(\bigvee\left\{\bigvee_{i \in J} A_{i}(x)\right\}, \bigwedge\left\{\bigwedge_{i \in J} B_{i}(x)\right\}\right) \text { for all } x \in f^{-1}(y) \\
& =\left(\bigvee_{i \in J} \bigvee\left\{A_{i}(x)\right\}, \bigwedge_{i \in J} \bigwedge\left\{B_{i}(x)\right\}\right) \text { for all } x \in f^{-1}(y) \\
& =\bigcup_{i \in J}\left(\bigvee\left\{A_{i}(x)\right\}, \bigwedge\left\{B_{i}(x)\right\}\right) \text { for all } x \in f^{-1}(y) \\
& =\bigcup_{i \in J}\left(f\left(A_{i}\right)(y), \overline{f\left(\overline{B_{i}}\right)}(y)\right)=\bigcup_{i \in J}\left(f\left(P_{i}\right)\right)(y) .
\end{aligned}
$$


(2) Let $P_{i}=\left(A_{i}, B_{i}\right)$ where $A_{i}, B_{i}$ are fuzzy rough sets for all $i \in J$. Then

$$
\begin{aligned}
& f\left(\bigcap_{i \in J} P_{i}\right)(y) \\
& \left.=f\left(\left(\bigcap_{i \in J} A_{i}, \bigcup_{i \in J} B_{i}\right)\right)(y)=\left(f\left(\bigcap_{i \in J} A_{i}\right)(y), \overline{f \overline{\left(\bigcup_{i \in J} B_{i}\right.}}\right)(y)\right) \\
& =\left(\bigvee\left\{\bigwedge_{i \in J} A_{i}(x)\right\}, \bigwedge\left\{\bigvee_{i \in J} B_{i}(x)\right\}\right) \text { for all } x \in f^{-1}(y) \\
& \leq\left(\bigwedge_{i \in J} \bigvee\left\{A_{i}(x)\right\}, \bigvee_{i \in J} \bigwedge\left\{B_{i}(x)\right\}\right) \text { for all } x \in f^{-1}(y) \\
& =\bigcap_{i \in J}\left(\bigvee\left\{A_{i}(x)\right\}, \bigwedge\left\{B_{i}(x)\right\}\right) \text { for all } x \in f^{-1}(y) \\
& =\bigcap_{i \in J}\left(f\left(A_{i}\right)(y), \overline{f\left(\overline{B_{i}}\right)}(y)\right)=\bigcap_{i \in J}\left(f\left(P_{i}\right)\right)(y) .
\end{aligned}
$$

\section{Conclusion}

The properties of the intuitionistic fuzzy rough sets are very complicated and inadequate in the sense of the extension of intuitionistic properties. This is because of the unnaturalness of the definition of fuzzy rough sets. Hence this flaw is critical in expanding the related theory. In order to overcome this unnaturalness, we introduce a new definition of intuitionistic fuzzy rough sets and investigate important properties about the image and inverse image of an intuitionistic rough sets under a mapping. This new approach enables us to manipulate fuzzy rough sets more simply and easily. All the results obtained from this new definition are different from the results in other papers, and will be proven useful in expanding the related theory.

\section{Conflict of Interest}

No potential conflict of interest relevant to this article was reported.

\section{References}

[1] L. A. Zadeh, "Fuzzy sets," Information and Control, vol. 8, no. 3, pp. 338-353, 1965. https://doi.org/10.1016/S00199958(65)90241-X

[2] Z. Pawlak, "Rough sets," International Journal of Computer \& Information Sciences, vol. 11, no. 5, pp. 341-356, 1982. https://doi.org/10.1007/BF01001956

[3] S. Nanda and S. Majumdar, "Fuzzy rough sets," Fuzzy Sets and Systems, vol. 45, no. 2, pp. 157-160, 1992. https: //doi.org/10.1016/0165-0114(92)90114-J
[4] D. Coker, "Fuzzy rough sets are intuitionistic L-fuzzy sets," Fuzzy Sets and Systems, vol. 96, no. 3, pp. 381-383, 1998. https://doi.org/10.1016/S0165-0114(97)00249-2

[5] K. T. Atanassov, "Intuitionistic fuzzy sets," Fuzzy Sets and Systems, vol. 20, no. 1, pp. 87-96, 1986. https://doi. org/10.1016/S0165-0114(86)80034-3

[6] S. K. Samanta and T. K. Mondal, "Intuitionistic fuzzy rough sets and rough intuitionistic fuzzy sets," Journal of Fuzzy Mathematics, vol. 9, no. 3, pp. 561-582, 2001.

[7] S. J. Lee and J. T. Kim, "Some properties of intuitionistic fuzzy bitopological spaces," in Proceedings of the 6th International Conference on Soft Computing and Intelligent Systems, and the 13th International Symposium on Advanced Intelligence Systems, Kobe, Japan, 2012, pp. 10401045. https://doi.org/10.1109/SCIS-ISIS.2012.6505156

[8] J. T. Kim and S. J. Lee, "Intuitionistic smooth bitopological spaces and continuity," International Journal of Fuzzy Logic and Intelligent Systems, vol. 14, no. 1, pp. 49-56, 2014. https://doi.org/10.5391/IJFIS.2014.14.1.49

[9] S. J. Lee and E. P. Lee, "The category of intuitionistic fuzzy topological spaces," Bulletin of the Korean Mathematical Society, vol. 37, no. 1, pp. 63-76, 2000.

[10] S. J. Lee and J. M. Chu, "Categorical property of intuitionistic topological spaces," Communications of the Korean Mathematical Society, vol. 24, no. 4, pp. 595-603, 2009. https://doi.org/10.4134/CKMS.2009.24.4.595

[11] J. T. Kim and S. J. Lee, "Categorical aspects of intuitionistic fuzzy topological spaces," International Journal of Fuzzy Logic and Intelligent Systems, vol. 15, no. 2, pp. 137-144, 2015. https://doi.org/10.5391/IJFIS.2015.15.2. 137

[12] K. Chakrabarty, R. Biswas, and S. Nanda, "Fuzziness in rough sets," Fuzzy Sets and Systems, vol. 110, no. 2, pp. 247-251, 2000. https://doi.org/10.1016/S0165-0114(97) 00414-4

[13] W. Z. Wu, J. S. Mi, and W. X. Zhang, "Generalized fuzzy rough sets," Information Sciences, vol. 151, pp. 263-282, 2003. https://doi.org/10.1016/S0020-0255(02)00379-1

[14] L. Zhou, W. Z. Wu, and W. X. Zhang, “On intuitionistic fuzzy rough sets and their topological structures," International Journal of General Systems, vol. 38, no. 6, pp. 589616, 2009. https://doi.org/10.1080/03081070802187723 
[15] L. Zhou, W. Z. Wu, and W. X. Zhang, "On characterization of intuitionistic fuzzy rough sets based on intuitionistic fuzzy implicators," Information Sciences, vol. 179, no. 7, pp. 883-898, 2009. https://doi.org/10.1016/j.ins.2008.11. 015

[16] X. Yang and Y. Yang, "Independence of axiom sets on intuitionistic fuzzy rough approximation operators," International Journal of Machine Learning and Cybernetics, vol. 4, no. 5, pp. 505-513, 2013. https://doi.org/10.1007/ s13042-012-0116-6

[17] S. M. Yun and S. J. Lee, "intuitionistic fuzzy approximation spaces induced by intuitionistic fuzzy topologies," in Proceedings of 2016 Joint 8th International Conference on Soft Computing and Intelligent Systems (SCIS) and 17th International Symposium on Advanced Intelligent Systems (ISIS), Sapporo, Japan, 2016, pp. 774-777. https://doi.org/10.1109/SCIS-ISIS.2016.0168

[18] S. M. Yun and S. J. Lee, "Intuitionistic fuzzy rough approximation operators," International Journal of Fuzzy Logic and Intelligent Systems, vol. 15, no. 3, pp. 208-215, 2015. https://doi.org/10.5391/IJFIS.2015.15.3.208
[19] S. M. Yun and S. J. Lee, "Intuitionistic fuzzy topologies induced by intuitionistic fuzzy approximation spaces," International Journal of Fuzzy Systems, vol. 19, no. 2, pp. 285291, 2017. https://doi.org/10.1007/s40815-016-0237-2

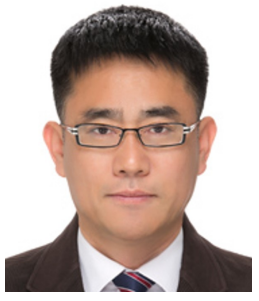

Sang Min Yun received his Ph.D. degree from Chungbuk National University in 2015. His research interests include general topology and fuzzy topology. He is a member of KIIS and KMS.

E-mail: jivesm@naver.com

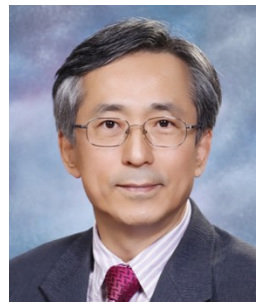

Seok Jong Lee received his M.S. and Ph.D. degrees from Yonsei University in 1986 and 1990 , respectively. He is a professor at the Department of Mathematics, Chungbuk National University since 1989. He was a visiting scholar in Carleton University from 1995 to 1996, and Wayne State University from 2003 to 2004. His research interests include general topology and fuzzy topology. He is a member of KIIS and KMS.

E-mail: sjl@cbnu.ac.kr 